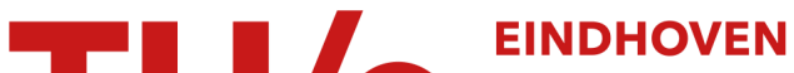 \\ UNIVERSITY OF \\ TECHNOLOGY
}

\section{Entangled photon pair generation from an InP membrane micro-ring resonator}

Citation for published version (APA):

Kumar, R. R., Raevskaia, M., Pogoretskii, V. G., Jiao, Y., \& Tsang, H. K. (2019). Entangled photon pair generation from an InP membrane micro-ring resonator. Applied Physics Letters, 114(2), [021104].

https://doi.org/10.1063/1.5080397

DOI:

$10.1063 / 1.5080397$

Document status and date:

Published: $14 / 01 / 2019$

Document Version:

Accepted manuscript including changes made at the peer-review stage

Please check the document version of this publication:

- A submitted manuscript is the version of the article upon submission and before peer-review. There can be important differences between the submitted version and the official published version of record. People interested in the research are advised to contact the author for the final version of the publication, or visit the $\mathrm{DOI}$ to the publisher's website.

- The final author version and the galley proof are versions of the publication after peer review.

- The final published version features the final layout of the paper including the volume, issue and page numbers.

Link to publication

\section{General rights}

Copyright and moral rights for the publications made accessible in the public portal are retained by the authors and/or other copyright owners and it is a condition of accessing publications that users recognise and abide by the legal requirements associated with these rights.

- Users may download and print one copy of any publication from the public portal for the purpose of private study or research.

- You may not further distribute the material or use it for any profit-making activity or commercial gain

- You may freely distribute the URL identifying the publication in the public portal.

If the publication is distributed under the terms of Article $25 \mathrm{fa}$ of the Dutch Copyright Act, indicated by the "Taverne" license above, please follow below link for the End User Agreement:

www.tue.nl/taverne

Take down policy

If you believe that this document breaches copyright please contact us at:

openaccess@tue.nl

providing details and we will investigate your claim. 


\section{Entangled photon pair generation from an InP membrane micro-ring resonator}

Cite as: Appl. Phys. Lett. 114, 021104 (2019); https://doi.org/10.1063/1.5080397

Submitted: 08 November 2018. Accepted: 03 January 2019. Published Online: 15 January 2019

Rakesh Ranjan Kumar (D), Marina Raevskaia, Vadim Pogoretskii, Yuqing Jiao (D), and Hon Ki Tsang (D)
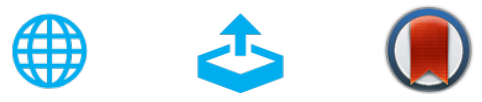

View Online

Export Citation

\section{ARTICLES YOU MAY BE INTERESTED IN}

Enhanced quantum dots spontaneous emission with metamaterial perfect absorbers Applied Physics Letters 114, 021103 (2019); https://doi.org/10.1063/1.5081688

High-power, high-spectral-purity GaSb-based laterally coupled distributed feedback lasers with metal gratings emitting at $2 \mu \mathrm{m}$

Applied Physics Letters 114, 021102 (2019); https://doi.org/10.1063/1.5080266

A simple approach to fiber-based tunable microcavity with high coupling efficiency Applied Physics Letters 114, 021106 (2019); https://doi.org/10.1063/1.5083011

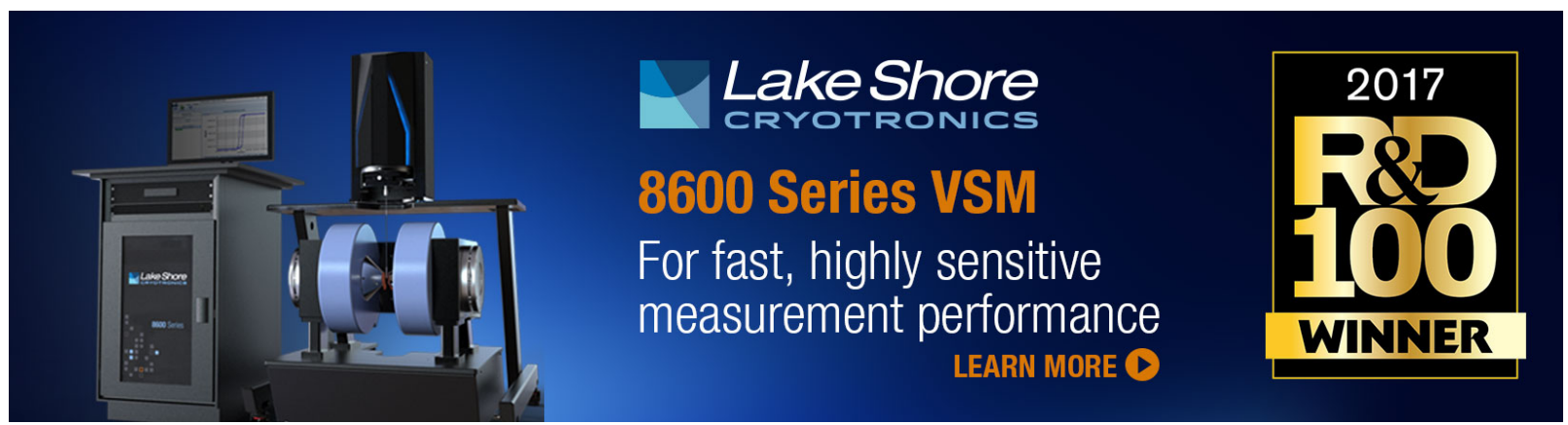




\title{
Entangled photon pair generation from an InP membrane micro-ring resonator
}

\author{
Cite as: Appl. Phys. Lett. 114, 021104 (2019); doi: 10.1063/1.5080397 \\ Submitted: 08 November 2018 - Accepted: 03 January 2019 • Published Online: \\ 15 January 2019
}

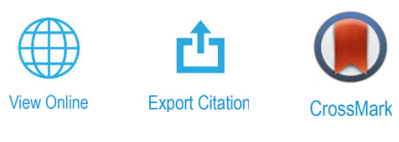

Rakesh Ranjan Kumar, (D) Marina Raevskaia, ${ }^{2}$ Vadim Pogoretskii, ${ }^{2}$ Yuqing Jiao, ${ }^{2}$ (D) and Hon Ki Tsang ${ }^{1, a)}$ (D)

\author{
AFFILIATIONS \\ ${ }^{7}$ Department of Electronic Engineering, The Chinese University of Hong Kong, Shatin, NT, Hong Kong \\ ${ }^{2}$ Institute for Photonic Integration (IPI), Eindhoven University of Technology, Eindhoven 5600 MB, The Netherlands \\ a) Electronic mail: hktsang@ee.cuhk.edu.hk.
}

\begin{abstract}
We performed quantum interference measurements using photon pairs generated via cavity enhanced spontaneous four-wave mixing in an indium phosphide membrane micro-ring resonator which was optically pumped by a continuous-wave laser. The internal generation rate was estimated to be as high as $70 \mathrm{kHz}$ at a coupled input power of 0.022 mW, and a two-photon quantum interference raw visibility of $78.4 \pm 2 \%$ was observed.
\end{abstract}

Published under license by AIP Publishing. https://doi.org/10.1063/1.5080397

Emerging applications in quantum communications, ${ }^{1}$ quantum computing, and quantum metrology ${ }^{3}$ have attracted increasing interest in the development of quantum photonics on different platforms for photonic integrated circuits, including silicon, silica, III-V semiconductors, and lithium niobate. ${ }^{4}$ The key functionalities of these processes are commonly realized from a pair of entangled single photon sources. Spontaneous four-wave mixing (SFWM) is an attractive process for generating a pair of entangled photons. ${ }^{4}$ Silicon waveguides and resonators have been widely used for SFWM because of their strong nonlinearity, scalability, CMOS compatibility, and mature fabrication technologies. ${ }^{4,5}$ While indium phosphide (InP) based photonic integrated circuits are well established for lasers, modulators, optical switches, and receivers, ${ }^{6}$ their use for the generation of entangled photons by SFWM has not been explored previously. InP offers higher nonlinearity than silicon, and the high index contrast of InP on benzocyclobutene (BCB) enables the fabrication of high quality-factor and small volume nonlinear micro-ring resonators ${ }^{6}$ which may be suitable for photon pair generation by SFWM. There is, however, a large two-photon absorption (TPA) coefficient in InP $(14.6 \mathrm{~cm} / \mathrm{GW}$ at $1.53 \mu \mathrm{m})$ and free carrier absorption $(\mathrm{FCA})^{7}$ that may hinder their suitability for photon pair generation.

In this paper, we experimentally assess the suitability of an $\mathrm{InP}$ ring resonator on $\mathrm{BCB}$ for generating photon pairs. Compared with silicon, the InP resonators require about ten times lower input power because of their higher third order nonlinear susceptibility.
The InP membrane waveguide schematic is shown in Fig. 1(a): the device is a side coupled ring resonator with a radius of $24 \mu \mathrm{m}$ coupled to a single bus waveguide with a designed gap between the ring and the bus waveguide of $300 \mathrm{~nm}$. The ring and bus waveguide have the same cross-sectional dimensions $(400 \times 300 \mathrm{~nm})$ and are integrated on a BCB/silicon carrier as illustrated in Fig. 1(a). The device fabrication method is described in Ref. 6. Single mode optical fibers were used for in-/out-coupling via waveguide grating couplers which have losses of about 6-dB per fiber-waveguide interface and a center wavelength at $1490 \mathrm{~nm}$. The micro-ring resonator used in the experiment is shown in Fig. 1(a), and its transmission spectrum, measured by a CW tunable laser (Keysight 81606A) set to an input power of 0.01 $\mathrm{mW}$, is recorded and shown in Fig. 2(a). The free spectral range of the device is $\sim 3.1 \mathrm{~nm}(421 \mathrm{GHz})$, and the three consecutive resonant wavelengths selected for SFWM to satisfy the energy and momentum conservation are $\lambda_{\mathrm{s}} \sim 1486.92 \mathrm{~nm}, \lambda_{\mathrm{p}} \sim 1490.05 \mathrm{~nm}$, and $\lambda_{\mathrm{i}} \sim 1493.16 \mathrm{~nm}$. The quality Q-factors (and the full width half maximum spectral width of the resonances) at these respective wavelengths are $43700\left(\Delta \lambda_{\mathrm{s}} \sim 0.034 \mathrm{~nm}\right), 45000\left(\Delta \lambda_{\mathrm{p}} \sim 0.033 \mathrm{~nm}\right)$, and $42700\left(\Delta \lambda_{\mathrm{i}} \sim 0.035 \mathrm{~nm}\right)$, from which we infer the cavity photon lifetime to be about $36 \mathrm{ps}$. The pump laser spectrum was filtered to suppress the background noise from amplified spontaneous emission (ASE) to achieve more than $>140 \mathrm{~dB}$ out of band suppression in the setup. The pump power was controlled by a variable optical attenuator (VOA), and the coupled power was monitored via a $1 \%$ tap coupler. The resonance wavelength of 


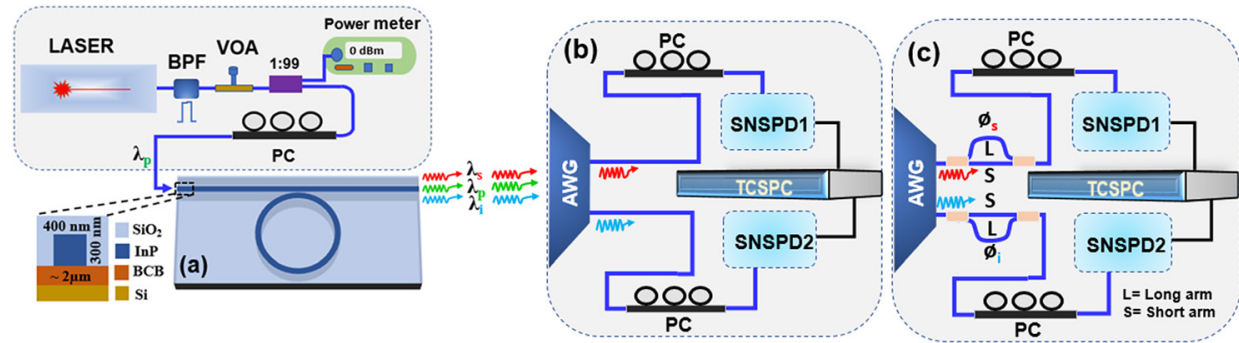

FIG. 1. Experimental setup: BPF: bandpass filter and VOA: variable optical attenuator. (a) Waveguide schematic and the InP membrane waveguide micro-ring resonator. (b) Correlated photon-pair measurements: AWG: arrayed waveguide grating, PC: polarization controller, and SNSPD: superconducting nanowire single photon detector. (c) Twophoton quantum interference measurement upon introducing two Franson interferometers.

the micro-ring resonator was precisely controlled by a thermoelectric Peltier cooler to prevent the resonance thermal drift caused by environmental temperature changes throughout the measurements. Each time the pump power was altered in Fig. 2(b), fine tuning of the laser wavelength to match the resonance wavelength was carried out. The generated signal/idler from the micro-ring was spectrally separated by two arrayed waveguide gratings (AWGs), which had a total insertion loss of about 7-dB

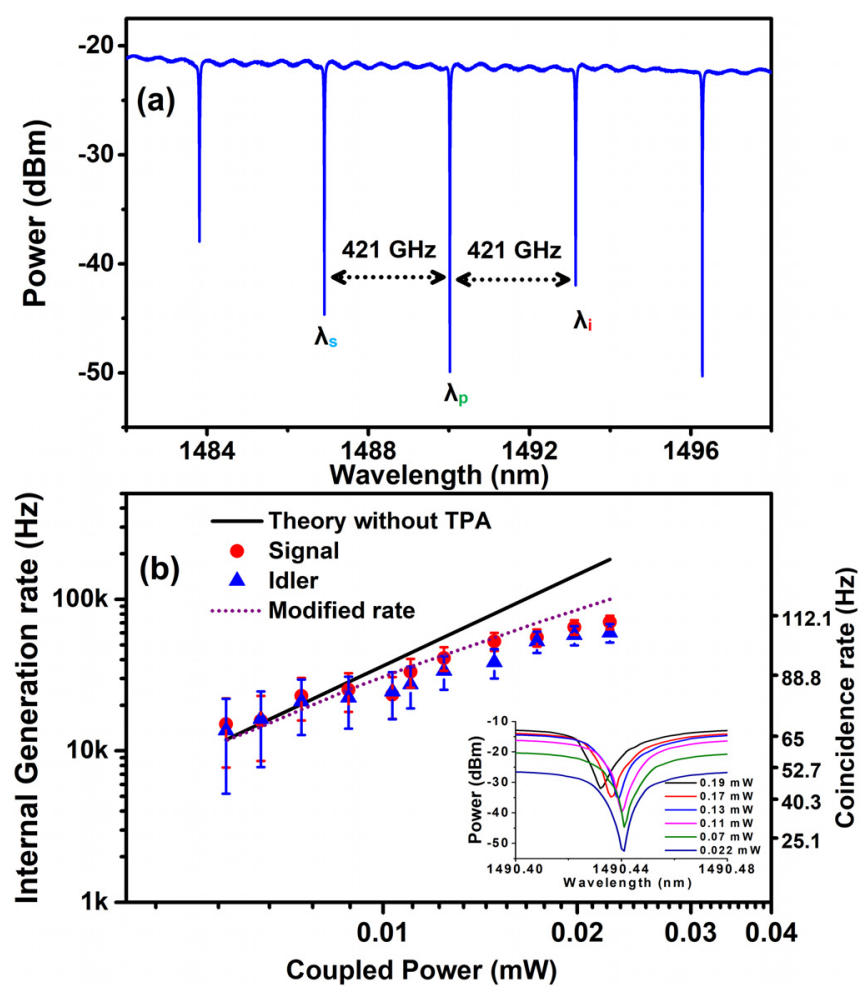

FIG. 2. (a) Transmission spectrum of the InP membrane waveguide ring resonator and (b) scaling of the internal generation rate of the signal (red circle) and the idler (blue triangle) generated through SFWM as a function of coupled power by considering the coupling loss, the filter insertion loss, and the detector quantum efficiency; the curves shown in the inset show the blueshift in resonance at higher pump power. The right-side vertical $y$-axis shows the raw measured coincidence rates. and which rejected the pump power by over $100 \mathrm{~dB}$. We estimate the total loss of $13 \mathrm{~dB}$ and $15 \mathrm{~dB}$ on signal and idler channels from the ring to the output of the AWG filter, including the 6-dB coupler loss of the output waveguide-fiber interface.

The internal generation rate at signal and idler wavelengths is estimated using Eq. (1) ${ }^{5}$ and plotted in Fig. 2(b)

$$
\mathrm{R}_{c}=(\gamma 2 \pi \mathrm{R})^{2}\left(\frac{\mathrm{Q} v_{g}}{\pi \omega_{p} \mathrm{R}}\right)^{3} \frac{v_{g}}{4 \pi \mathrm{R}} \mathrm{P}_{p}{ }^{2},
$$

where $\mathrm{R}, \mathrm{P}_{\mathrm{p}}, \mathrm{Q}, \omega_{\mathrm{p}}$, and $\mathrm{v}_{\mathrm{g}}$ are the radius of the ring, the coupled pump power, the quality factor, the angular frequency, and the group velocity at the pump wavelength $\mathrm{v}_{\mathrm{g}}=6.25 \times 10^{7} \mathrm{~m} / \mathrm{s}$. The nonlinear coefficient $\gamma=1590 \mathrm{~W}^{-1} \mathrm{~m}^{-1}$ was calculated from the nonlinear effective index $\mathrm{n}_{2}=2.7 \times 10^{-17} \mathrm{~m}^{2} / \mathrm{W}$. Taking into account the output grating coupler loss and the AWG filter loss, the internal generation rate is calculated to be $\sim 70 \mathrm{kHz}$, limited by the nonlinear losses at a coupled power of $0.022 \mathrm{~mW}$ as shown in Fig. 2(b). The on-chip pair generation rate (PGR) from Eq. (1) may be expressed as PGR $=\mathrm{g} \times \mathrm{P}^{2}$, where $\mathrm{g}\left(\mathrm{MHz} / \mathrm{mW}^{2}\right)$ is the PGR coefficient. In the fitted line in Fig. 2(b), $g=145 \mathrm{MHz} /$ $\mathrm{mW}^{2}$, which is comparable to the results $\left(\mathrm{g}=149 \mathrm{MHz} / \mathrm{mW}^{2}\right.$ ) obtained by Ma et al. using a silicon micro-ring resonator with double the $\mathrm{Q}$ and less than half the radius of the InP device. ${ }^{8}$

Notice that Eq. (1) does not account for TPA, ${ }^{9}$ and experimental data in Fig. 2(b) at higher coupled powers show deviations from Eq. (1) due to larger TPA in InP. At a coupled power above $0.022 \mathrm{~mW}$, large TPA and the associated net decrease in the refractive index produced by the free carrier dispersion ${ }^{10}$ in the InP micro-ring resonator result in an experimentally observed blueshift in the resonance as shown in the inset of Fig. 2(b). However, nonlinear losses that arise from the TPA and the FCA in the cavity are considered, and the modified count rate is plotted in the dashed curve in Fig. 2(b) based on the analysis discussed by Guo et al. ${ }^{11}$ The comparison shows that Eq. (1) does not follow the experimental data with increasing pump power, but consideration of nonlinear losses in the cavity under high pump power fits much better with the experimental data. The experimental data have signal and idler measurement error bars equal to the standard deviation of the raw signal and the idler count rate.

The experimental results were obtained using two superconducting nanowire single photon detectors (SNSPDs). The 
quantum efficiency of the detectors is above $70 \%$, and the time jitter is less than $50 \mathrm{ps}$. The time correlated single photon counting electronics has a time jitter of less than $30 \mathrm{ps}$. The exact instant of a photon pair emission from the continuous wave (CW) pump laser is uncertain within the coherence time of the pump laser. Therefore, two photons' relative arrival time differences are plotted as a histogram and are shown in Fig. 3(a), which map the arrival-time differences to different time-bins within the 20-min integration time window.

The histogram has a Gaussian profile, and the FWHM is consistent with the timing jitter of the single photon detector. We measured the FWHM of the distribution of arrival time differences to be $115 \mathrm{ps}$ and the coincidence to accidental ratio (CAR) to be 277 in Fig. 3(a), deduced from the experimental setup in Fig. 1(b). The FWHM of the coincidence histogram is limited by the time jitter of the detectors and the cavity photon lifetime of the signal and idler resonances to be $\sim 36 \mathrm{ps}$. The CAR value was calculated from the number of coincidences at the peak over the number of coincidences away from the peak in Fig. 3(a) on the same time window. The CAR value was measured at the highest coupled power of $0.022 \mathrm{~mW}$. We experimentally measured the two-photon quantum interference, using two Franson interferometers which had path length differences that satisfy the conditions described in Ref. 12 as shown in Fig. 1(c). The output of the Franson interferometers was measured by two SNSPDs, and the arrival time differences are plotted in the histograms of Fig. 3(b), which has a central peak produced when pairs of photons both travel the long arms or both travel the short arm of the Franson interferometers. The path followed by the bi-photon states for the central peak is indistinguishable, while the other two side peaks are produced when one photon travels the short path and the other photon travels the long path and vice versa. The side peaks allow the paths travelled to be

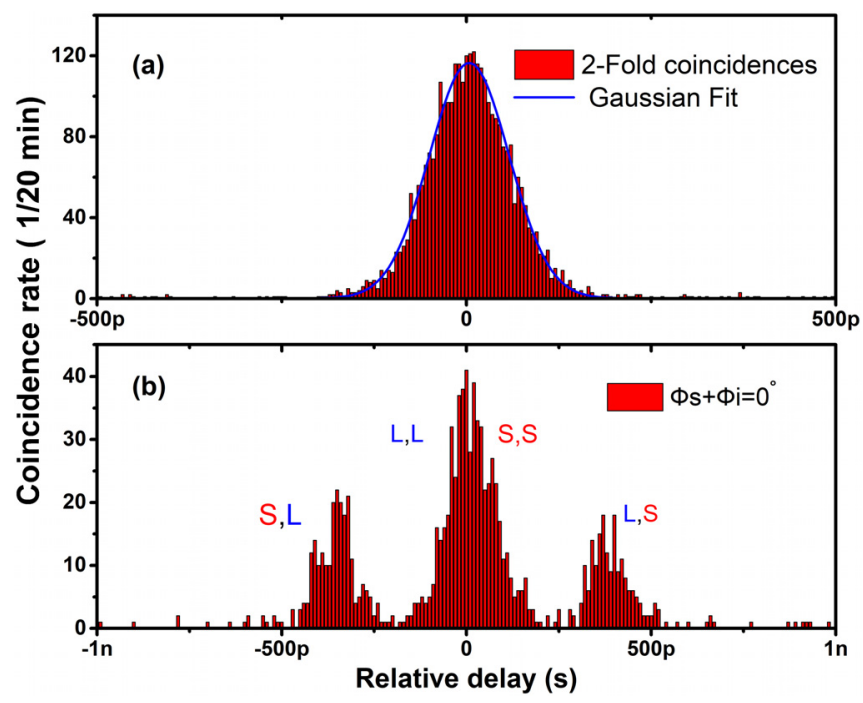

FIG. 3. (a) Time-bin coincidence histogram of the arrival of pair photons and (b) time-bin coincidence histogram measured at the output of the interferometers integrated over $20 \mathrm{~min}$ at a coupled pump power of $0.022 \mathrm{~mW}$. distinguishable. The path imbalanced $(\Delta \mathrm{T})$ arm of the interferometer must be larger than the coherence time of bi-photons $(\tau)$ to prevent the first order interference,${ }^{12}$ the absence of which was validated from the experimental results shown in Fig. 4(c). The interferometer used in this experiment has $\Delta \mathrm{T} \sim 0.4 \mathrm{~ns}$ and $\tau \sim 36$ ps (measured from the linewidth of the resonances modes), which satisfies the condition proposed in Ref. 12. The absence of the single count variation of the signal and the idler with the interferometer phase shown in Fig. 4(c) demonstrates that the interferometer imbalance time is much greater than the emission time uncertainty of the pairs of photons. The two fiber interferometers were mounted on Peltier stages separately, which were controlled by a precise identical temperature controller to control the phase of the individual interferometer. The two-photon interference visibility can be calculated from the measured coincidence count rates between single and idler photons while varying the phase of both interferometers. Therefore, we measured the coincidence probability of pair of photons which is expected to be $\mathrm{be}^{13,14}$
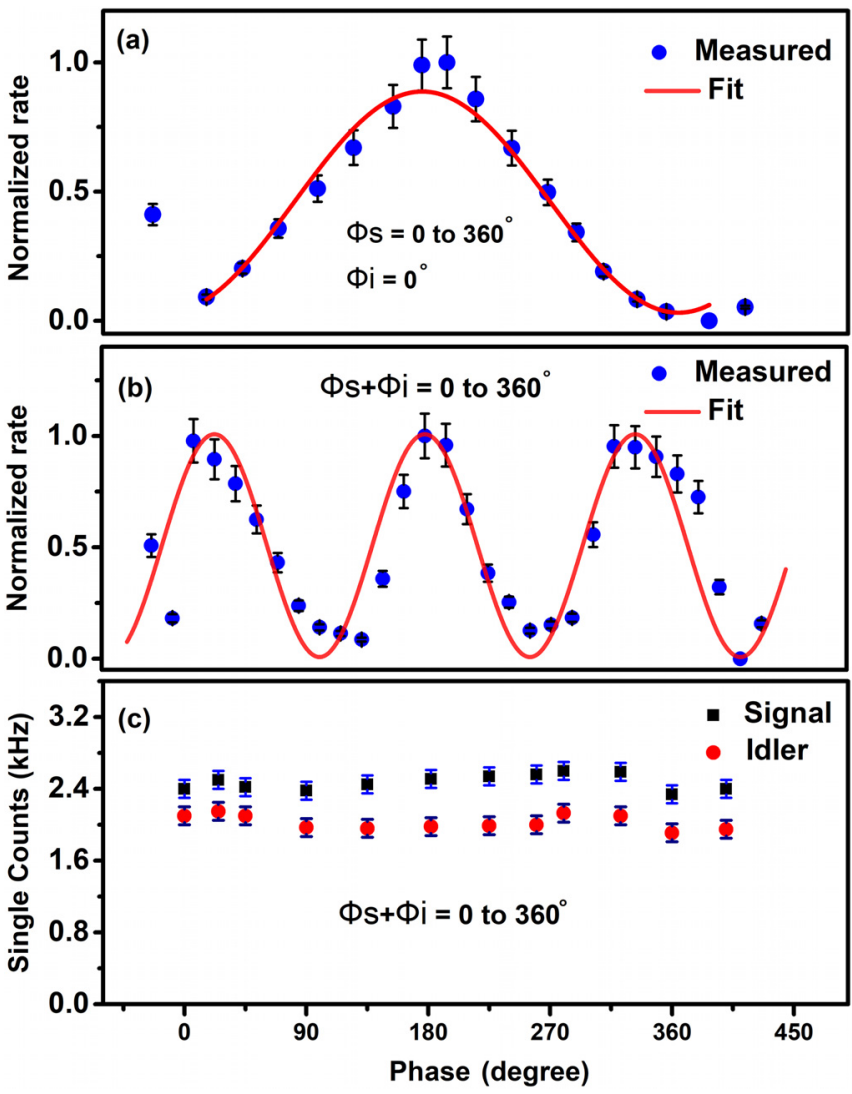

FIG. 4. (a) Only the signal interferometer phase $\left(\emptyset_{s}\right)$ was changed. The error bars indicate the standard deviation on the count rate. The integration time of each point is $120 \mathrm{~s}$. (b) Both signal and idler interferometer phases $\left(\varnothing_{\mathrm{s}}+\varnothing_{\mathrm{i}}\right)$ were changed. The integration time of each point is $180 \mathrm{~s}$. The error bars indicate the standard deviation of the count rate. (c) The complete absence of interference in the single count of the signal and the idler validating our interferometer coherence time is much larger than the bi-photon generated coherence time. 


$$
\mathbf{C}\left(\varnothing_{s}+\varnothing_{i}\right) \propto \cos \left(\varnothing_{s}+\varnothing_{i}+\boldsymbol{\delta}\right)
$$

where $\emptyset_{\mathrm{s}}$ and $\emptyset_{\mathrm{i}}$ are the phase of the signal and the idler and $\delta$ is a constant phase term.

In the first measurement, only the signal interferometer phase $\left(\emptyset_{\mathrm{s}}\right)$ was changed as shown in Fig. 4(a), whereas in the second measurement, both the signal and idler interferometer phases were changed in Fig. 4(b) where each point was recorded at an integration time of $180 \mathrm{~s}$. We observed the raw two-photon quantum interference visibilities of $\mathrm{V}_{\text {meas }}=78.4 \pm 2 \%$, which is greater than $\sim 71 \%$, thus confirming the entanglement through violation of Bell's inequality ${ }^{15}$ at a coupled power of $0.022 \mathrm{~mW}$. The measured visibility defined as $V_{\text {meas }}=\left(\mathrm{N}_{\max }-\mathrm{N}_{\min }\right) /\left(\mathrm{N}_{\max }\right.$ $+\mathrm{N}_{\text {min }}$ ), where $\mathrm{N}_{\max }$ and $\mathrm{N}_{\min }$ represent the maxima and minima in Fig. 4(b), obtained from the best fit of the experimental data and error on the visibility, is measured from the standard deviation of three sets of experimental data.

We also included the effect on two photon coincidence count rates shown in Figs. 5(a)-5(d) with the phase angle, where middle peaks correspond to indistinguishable photon arrival events, which are three times larger than the other two side peaks which are distinguishable photon uncorrelated events that appear to be independent of phases $\emptyset_{\mathrm{s}}$ and $\emptyset_{\mathrm{i}}$. We only show four coincidence rate plots in Figs. 5(a)-5(d) corresponding to the different interferometer phase angle in the experimental result shown in Fig. 4(b). The middle peak coincidence rate variation with the phase was validated in the measurement in Fig. 4(b) and fitted by a sinusoidal curve, which followed Eq. (2) and

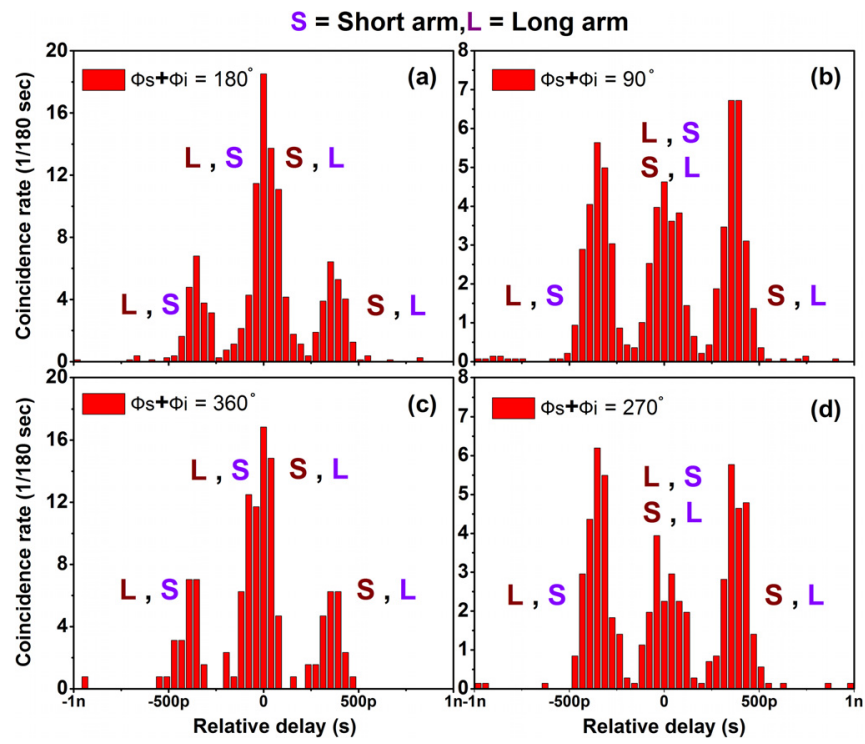

FIG. 5. (a) Coincidence count rate was measured when both interferometer phases were set to $\emptyset_{\mathrm{s}}+\emptyset_{\mathrm{i}}=180^{\circ}$ analogous to the middle peak in Fig. $4(\mathrm{~b})$. (b) When both interferometer phases were set to $\emptyset_{\mathrm{s}}+\emptyset_{\mathrm{i}}=90^{\circ}$, the middle peak coincidence count rate should be zero, but the small number comes from the accidental background count rate from uncorrelated photons. (c) Coincidence count rate was measured when both interferometers set to $\emptyset_{\mathrm{s}}+\emptyset_{\mathrm{i}}=360^{\circ}$ correspond to the rightmost peak in Fig. 4 (b). (d) When both interferometers set to $\emptyset_{\mathrm{s}}+\emptyset_{\mathrm{i}}=270^{\circ}$ represent the trough in Fig. $4($ b). confirmed the two-photon quantum interference and the entanglement from violation of the Bell inequality. ${ }^{15}$

The observations of entangled photon pair generation from InP membrane waveguide resonators show the potential advantages and limitations of the high nonlinearity InP platform. The experiment showed that while SFWM is efficient in generating entangled photons, there were limits to its scalability to higher pump powers because of nonlinear loss which limits the purity of single photon emission reported in the same device used here. ${ }^{16}$ By limiting the pump power to $0.022 \mathrm{~mW}$, it was nonetheless possible to produce high-quality biphotons by taking advantage of the cavity enhanced from the ring resonator. ${ }^{17}$ Thus, the bi-photon state reaching the detector is denoted as $|\psi\rangle=\frac{1}{\sqrt{2}}\left(|s, s\rangle+e^{i \varphi}|l, l\rangle\right)$, where $\mathrm{s}$ and 1 denote the short and long arm lengths in our interferometer and $\varnothing=\emptyset_{i}+\emptyset_{\mathrm{s}}$ are phases of signal and idler interferometers. Hence, we expect to see constructive to destructive interference which will be twice that of one photon rate shown in Fig. 4(a) with the change in the phase of the signal channel, whereas the idler phase was constant and compared with Fig. 4(b), validating the measurement as a two-photon quantum interference with half period of the one photon interference. Finally, the relatively poor visibility in this experiment was caused by experimental limitations including fine shifts in resonance at different powers (reducing the efficiency of generated photons) and differences in the path imbalance arm $(\Delta \mathrm{T})$ of Franson interferometers. In order to maximize the visibility, the imbalance in the interferometer of the signal and the idler must be equal in order to provide the maximum overlap when both photons take the long path or when both photons take the short path. ${ }^{18,19}$ However, the Franson interferometer used here in the experiment was based on a fiber interferometer made by fiber splicing and without any free space tunable optical delay. The ratio of delay of both fiber interferometers was measured to be $\Delta \mathrm{T}_{\mathrm{s}} / \Delta \mathrm{T}_{\mathrm{i}}=1.076$, which introduces a systematic delay imbalance of $7.6 \%$. The amplitude of oscillation of the biphoton state will be limited by temporal overlap offset from the delay imbalance, which leads to both a decrease in the coincidence count rate and the visibility. Many results tabulated in Table I use the free space optic Franson interferometer, where both imbalance $\operatorname{arms}(\Delta \mathrm{T})$ can be precisely tuned to be equal, 8,14,20,21 and increases the visibility compared to this work. Thus, the measurement has only a modest visibility level of $\mathrm{V}_{\text {meas }} \sim 78.4 \pm 2 \%$.

In conclusion, we experimentally demonstrated the twophoton quantum interference using an InP membrane micro-

TABLE I. Comparison between silicon micro-ring resonators as an entangled photon pair source.

\begin{tabular}{lccccc}
\hline \hline References & Radius $(\mu \mathrm{m})$ & $\mathrm{Q}$ & PGR coefficient $\left(\mathrm{MHz} \mathrm{mW}^{-2}\right)$ & $\mathrm{CAR}$ & $\mathrm{V}^{\mathrm{a}}(\%)$ \\
\hline 8 & 10 & $9.2 \times 10^{4}$ & $\sim 149$ & 532 & 98 \\
14 & 7 & $2 \times 10^{4}$ & $\sim 129$ & 352 & 93 \\
20 & 10 & $1.5 \times 10^{4}$ & $\sim 7$ & 132 & 95 \\
21 & 10 & $2 \times 10^{4}$ & $\sim 40$ & 350 & 82 \\
This work & 24 & $4.2 \times 10^{4}$ & $\sim 145$ & 277 & 78 \\
\hline \hline
\end{tabular}

The measured visibility, as stated in the article. 
ring resonator for generating entangled photons. The microring resonator has been widely used for entangled photon generation in silicon and other materials and were studied extensively in the past for SFWM, ${ }^{4}$ but InP based micro-ring resonators have not been previously studied for SFWM based entangled photon generation. The source is capable of generating pair photons $(70 \mathrm{kHz})$ at sub-milliwatts $(0.022 \mathrm{~mW})$ coupled power, reducing the foot-print of the future quantum device compared to other reported works. $8,14,20,21$

This work was supported by the Hong Kong Research Grants Council, General Research fund (RGC, GRF) (14207117).

\section{REFERENCES}

${ }^{1}$ N. Gisin and R. Thew, Nat. Photonics 1, 165 (2007).

${ }^{2}$ D. Gottesman and I. L. Chuang, Nature 402, 390 (1999).

${ }^{3}$ V. Giovannetti, S. Lloyd, and L. Maccone, Nat. Photonics 5, 222 (2011).

4J. W. Silverstone, D. Bonneau, J. L. O' Brien, and M. G. Thomson, IEEE J. Sel. Top. Quantum Electron. 22, 390 (2016).

${ }^{5}$ S. Azzini, D. Grassani, M. J. Strain, M. Sorel, L. G. Helt, J. E. Sipe, M. Liscidini, M. Galli, and D. Bajoni, Opt. Express 20, 23100 (2012).

${ }^{6}$ J. Van der Tol, Y. Jiao, L. Shen, A. Millan-Mejia, V. Pogoretskii, J. P. van Engelen, and M. Smit, IEEE J. Sel. Top. Quantum Electron. 24, 1 (2018).
${ }^{7}$ M. Heuck, S. Combrie, G. Lehoucq, S. Malaguti, G. Bellanca, S. Trillo, P. T. Kristensen, J. Mork, J. P. Reithmaier, and A. de Rossi, Appl. Phys. Lett. 103, 181120 (2013).

${ }^{8}$ C. Ma, X. Wang, V. Anant, A. D. Beyer, M. D. Shaw, and S. Mookherjea, Opt. Express 25, 32995 (2017).

${ }^{9}$ C. A. Husko, A. S. Clark, M. J. Collins, A. D. Rossi, S. Combrie, G. Lehoucq, I. H. Rey, T. F. Krauss, C. Xiong, and B. J. Eggleton, Sci. Rep. 3, 3087 (2013).

${ }^{10}$ T. J. Johnson, M. Borselli, and O. Painter, Opt. Express 14, 817 (2006).

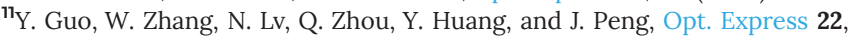
2620 (2014).

12 J. D. Franson, Phys. Rev. Lett. 62, 2205 (1989).

${ }^{13}$ K. Harada, H. Takesue, H. Fukuda, T. Tsuchizawa, T. Watanabe, K. Yamada, Y. Tokura, and S. Itabashi, Opt. Express 16, 20368 (2008).

${ }^{14}$ R. Wakabayashi, M. Fujiwara, K. Yoshino, Y. Nambu, M. Sasaki, and T. Aoki, Opt. Express 23, 1103 (2015).

${ }^{15}$ P. G. Kwiat, A. M. Steinberg, and R. Y. Chiao, Phys. Rev. A 47, R2472 (1993).

${ }^{16}$ R. R. Kumar, M. Feng, M. Raevskaia, V. Pogoretskii, Y. Jiao, and H. K. Tsang, in IEEE Photonics Conference (IPC), Reston, VA, October 2018.

${ }^{17}$ S. Clemmen, K. P. Huy, W. Bogaerts, R. Baets, Ph. Emplit, and S. Massar, Opt. Express 17, 16558 (2009).

${ }^{18}$ J.-P. W. Maclean, J. M. Donohue, and K. J. Resch, Phys. Rev. A. 97, 063826 (2018).

${ }^{19}$ W. P. Grice and I. A. Walmsley, Phys. Rev. A 56, 1627 (1997).

${ }^{20}$ D. Grassani, S. Azzini, M. Liscidini, M. Galli, M. J. Strain, M. Sorel, J. E. Sipe, and D. Bajoni, Optica 2, 88 (2015).

${ }^{21}$ M. Fujiwara, R. Wakabayashi, M. Sasaki, and M. Takeoka, Opt. Express 25, 3445 (2017). 